Proceedings of the Institute of Mathematics and Mechanics,

National Academy of Sciences of Azerbaijan

Volume 46, Number 2, 2020, Pages 197-209

https://doi.org/10.29228/proc. 29

\title{
SOME INEQUALITIES FOR WEIGHTED AND INTEGRAL MEANS OF CONVEX FUNCTIONS ON LINEAR SPACES
}

\author{
SILVESTRU SEVER DRAGOMIR
}

\begin{abstract}
Let $f$ be a convex function on a convex subset $C$ of a linear space and $x, y \in C$, with $x \neq y$. If $p:[0,1] \rightarrow \mathbb{R}$ is a Lebesgue integrable and symmetric function, namely $p(1-t)=p(t)$ for all $t \in[0,1]$ and such that the condition
\end{abstract}

$$
0 \leq \int_{0}^{\tau} p(s) d s \leq \int_{0}^{1} p(s) d s \text { for all } \tau \in[0,1]
$$

holds, then we have

$$
\begin{aligned}
& \left|\frac{1}{\int_{0}^{1} p(\tau) d \tau} \int_{0}^{1} p(\tau) f((1-\tau) x+\tau y) d \tau-\int_{0}^{1} f((1-\tau) x+\tau y) d \tau\right| \\
& \leq \frac{1}{\int_{0}^{1} p(\tau) d \tau} \int_{0}^{1}\left(\int_{0}^{\tau} p(s) d s\right)(1-\tau) d \tau\left[\nabla_{-} f_{y}(y-x)-\nabla_{+} f_{x}(y-x)\right] \\
& \leq \frac{1}{2}\left[\nabla_{-} f_{y}(y-x)-\nabla_{+} f_{x}(y-x)\right] .
\end{aligned}
$$

Some applications for norms and semi-inner products are also provided.

\section{Introduction}

Let $X$ be a real linear space, $x, y \in X, x \neq y$ and let

$$
[x, y]:=\{(1-\lambda) x+\lambda y, \lambda \in[0,1]\}
$$

be the segment generated by $x$ and $y$. We consider the function $f:[x, y] \rightarrow \mathbb{R}$ and the attached function $\varphi_{(x, y)}:[0,1] \rightarrow \mathbb{R}, \varphi_{(x, y)}(t):=f[(1-t) x+t y], t \in[0,1]$.

It is well known that $f$ is convex on $[x, y]$ iff $\varphi(x, y)$ is convex on $[0,1]$, and the following lateral derivatives exist and satisfy

(i) $\varphi_{ \pm(x, y)}^{\prime}(s)=\nabla_{ \pm} f_{(1-s) x+s y}(y-x), s \in[0,1)$,

(ii) $\varphi_{+(x, y)}^{\prime}(0)=\nabla_{+} f_{x}(y-x)$,

(iii) $\varphi_{-(x, y)}^{\prime}(1)=\nabla_{-} f_{y}(y-x)$,

2010 Mathematics Subject Classification. 26D15; 46B05.

Key words and phrases. Convex functions, Linear spaces, Integral inequalities, HermiteHadamard inequality, Féjer's inequalities, Norms and semi-inner products. 
where $\nabla_{ \pm} f_{x}(y)$ are the Gâteaux lateral derivatives, we recall that

$$
\begin{aligned}
& \nabla_{+} f_{x}(y):=\lim _{h \rightarrow 0+} \frac{f(x+h y)-f(x)}{h}, \\
& \nabla_{-} f_{x}(y):=\lim _{k \rightarrow 0-} \frac{f(x+k y)-f(x)}{k}, x, y \in X .
\end{aligned}
$$

The following inequality is the well-known Hermite-Hadamard integral inequality for convex functions defined on a segment $[x, y] \subset X$ :

$$
f\left(\frac{x+y}{2}\right) \leq \int_{0}^{1} f[(1-t) x+t y] d t \leq \frac{f(x)+f(y)}{2},
$$

which easily follows by the classical Hermite-Hadamard inequality for the convex function $\varphi(x, y):[0,1] \rightarrow \mathbb{R}$

$$
\varphi_{(x, y)}\left(\frac{1}{2}\right) \leq \int_{0}^{1} \varphi_{(x, y)}(t) d t \leq \frac{\varphi_{(x, y)}(0)+\varphi_{(x, y)}(1)}{2} .
$$

For other related results see the monograph on line [8]. For some recent results in linear spaces see [1], [2] and [9]-[12].

In the recent paper [7] we established the following refinements and reverses of Féjer's inequality for functions defined on linear spaces:

Theorem 1.1. Let $f$ be an convex function on $C$ and $x, y \in C$ with $x \neq y$. If $p:[0,1] \rightarrow[0, \infty)$ is Lebesgue integrable and symmetric, namely $p(1-t)=p(t)$ for all $t \in[0,1]$, then

$$
\begin{aligned}
0 & \leq \frac{1}{2}\left[\nabla_{+} f_{\frac{x+y}{2}}(y-x)-\nabla_{-} f_{\frac{x+y}{2}}(y-x)\right] \int_{0}^{1}\left|t-\frac{1}{2}\right| p(t) d t \\
& \leq \int_{0}^{1} f((1-t) x+t y) p(t) d t-f\left(\frac{x+y}{2}\right) \int_{0}^{1} p(t) d t \\
& \leq \frac{1}{2}\left[\nabla_{-} f_{y}(y-x)-\nabla_{+} f_{x}(y-x)\right]\left(\int_{0}^{1}\left|t-\frac{1}{2}\right| p(t) d t\right)
\end{aligned}
$$

and

$$
\begin{aligned}
0 & \leq \frac{1}{2}\left[\nabla_{+} f_{\frac{x+y}{2}}(y-x)-\nabla_{-} f_{\frac{x+y}{2}}(y-x)\right] \int_{0}^{1}\left(\frac{1}{2}-\left|t-\frac{1}{2}\right|\right) p(t) d t \\
& \leq \frac{f(x)+f(y)}{2} \int_{0}^{1} p(t) d t-\int_{0}^{1} f((1-t) x+t y) p(t) d t \\
& \leq \frac{1}{2}\left[\nabla_{-} f_{y}(y-x)-\nabla_{+} f_{x}(y-x)\right] \int_{0}^{1}\left(\frac{1}{2}-\left|t-\frac{1}{2}\right|\right) p(t) d t .
\end{aligned}
$$

If we take $p \equiv 1$ in (1.1), then we get

$$
\begin{aligned}
0 & \leq \frac{1}{8}\left[\nabla_{+} f_{\frac{x+y}{2}}(y-x)-\nabla_{-} f_{\frac{x+y}{2}}(y-x)\right] \\
& \leq \int_{0}^{1} f[(1-t) x+t y] d t-f\left(\frac{x+y}{2}\right) \\
& \leq \frac{1}{8}\left[\nabla_{-} f_{y}(y-x)-\nabla_{+} f_{x}(y-x)\right]
\end{aligned}
$$


that was firstly obtained in [4], while from (1.2) we recapture the result obtained in $[5]$

$$
\begin{aligned}
0 & \leq \frac{1}{8}\left[\nabla_{+} f_{\frac{x+y}{2}}(y-x)-\nabla_{-} f_{\frac{x+y}{2}}(y-x)\right] \\
& \leq \frac{f(x)+f(y)}{2}-\int_{0}^{1} f[(1-t) x+t y] d t \\
& \leq \frac{1}{8}\left[\nabla_{-} f_{y}(y-x)-\nabla_{+} f_{x}(y-x)\right] .
\end{aligned}
$$

Motivated by the above results, we establish in this paper some upper and lower bounds for the difference

$$
\int_{0}^{1} p(\tau) f((1-\tau) x+\tau y) d \tau-\int_{0}^{1} p(\tau) d \tau \int_{0}^{1} f((1-\tau) x+\tau y) d \tau
$$

where $f$ is a convex function on $C$ and $x, y \in C$, with $x \neq y$ while $p:[0,1] \rightarrow \mathbb{R}$ is a Lebesgue integrable function such that

$$
0 \leq \int_{0}^{\tau} p(s) d s \leq \int_{0}^{1} p(s) d s \text { for all } \tau \in[0,1] .
$$

Some applications for norms and semi-inner products are also provided.

\section{Main Results}

We start to the following identity that is of interest in itself as well:

Lemma 2.1. Let $f$ be a convex function on $C$ and $x, y \in C$, with $x \neq y$. If $g:[0,1] \rightarrow \mathbb{C}$ is a Lebesgue integrable function, then we have the equality

$$
\begin{aligned}
& \int_{0}^{1} g(\tau) \varphi_{(x, y)}(\tau) d \tau-\int_{0}^{1} g(\tau) d \tau \int_{0}^{1} \varphi_{(x, y)}(\tau) d \tau \\
& =\int_{0}^{1}\left(\int_{\tau}^{1} g(s) d s\right) \tau \varphi_{(x, y)}^{\prime}(\tau) d \tau \\
& +\int_{0}^{1}\left(\int_{0}^{\tau} g(s) d s\right)(\tau-1) \varphi_{(x, y)}^{\prime}(\tau) d \tau
\end{aligned}
$$

Proof. Integrating by parts in the Lebesgue integral, we have

$$
\begin{aligned}
& \int_{0}^{\tau} t \varphi_{(x, y)}^{\prime}(t) d t+\int_{\tau}^{1}(t-1) \varphi_{(x, y)}^{\prime}(t) d t \\
& =\tau \varphi_{(x, y)}(\tau)-\int_{0}^{\tau} \varphi_{(x, y)}(t) d t-(\tau-1) \varphi_{(x, y)}(\tau)-\int_{\tau}^{1} \varphi_{(x, y)}(t) d t \\
& =\varphi_{(x, y)}(\tau)-\int_{0}^{1} \varphi_{(x, y)}(t) d t
\end{aligned}
$$

that holds for all $\tau \in[0,1]$. 
If we multiply this identity by $g(\tau)$ and integrate over $\tau$ in $[0,1]$, then we get

$$
\begin{aligned}
& \int_{0}^{1} g(\tau) \varphi_{(x, y)}(\tau) d \tau-\int_{0}^{1} g(\tau) d \tau \int_{0}^{1} \varphi_{(x, y)}(t) d t \\
& =\int_{0}^{1} g(\tau)\left(\int_{0}^{\tau} t \varphi_{(x, y)}^{\prime}(t) d t\right) d \tau+\int_{0}^{1} g(\tau)\left(\int_{\tau}^{1}(t-1) \varphi_{(x, y)}^{\prime}(t) d t\right) d \tau .
\end{aligned}
$$

Using integration by parts, we get

$$
\begin{aligned}
& \int_{0}^{1} g(\tau)\left(\int_{0}^{\tau} t \varphi_{(x, y)}^{\prime}(t) d t\right) d \tau \\
= & \int_{0}^{1}\left(\int_{0}^{\tau} t \varphi_{(x, y)}^{\prime}(t) d t\right) d\left(\int_{0}^{\tau} g(s) d s\right) \\
= & \left.\left(\int_{0}^{\tau} g(s) d s\right)\left(\int_{0}^{\tau} t \varphi_{(x, y)}^{\prime}(t) d t\right)\right|_{0} ^{1} \\
& -\int_{0}^{1}\left(\int_{0}^{\tau} g(s) d s\right) \tau \varphi_{(x, y)}^{\prime}(\tau) d \tau \\
= & \left(\int_{0}^{1} g(s) d s\right)\left(\int_{0}^{1} t \varphi_{(x, y)}^{\prime}(t) d t\right) \\
- & \int_{0}^{1}\left(\int_{0}^{\tau} g(s) d s\right) \tau \varphi_{(x, y)}^{\prime}(\tau) d \tau \\
= & \int_{0}^{1}\left(\int_{0}^{1} g(s) d s-\int_{0}^{\tau} g(s) d s\right) \tau \varphi_{(x, y)}^{\prime}(\tau) d \tau \\
= & \int_{0}^{1}\left(\int_{\tau}^{1} g(s) d s\right) \tau \varphi_{(x, y)}^{\prime}(\tau) d \tau
\end{aligned}
$$

and

$$
\begin{aligned}
& \int_{0}^{1} g(\tau)\left(\int_{\tau}^{1}(t-1) \varphi_{(x, y)}^{\prime}(t) d t\right) d \tau \\
& =\int_{0}^{1}\left(\int_{\tau}^{1}(t-1) \varphi_{(x, y)}^{\prime}(t) d t\right) d\left(\int_{0}^{\tau} g(s) d s\right) \\
& =\left.\left(\int_{\tau}^{1}(t-1) \varphi_{(x, y)}^{\prime}(t) d t\right)\left(\int_{0}^{\tau} g(s) d s\right)\right|_{0} ^{1} \\
& +\int_{0}^{1}\left(\int_{0}^{\tau} g(s) d s\right)(\tau-1) \varphi_{(x, y)}^{\prime}(\tau) d \tau \\
& =\int_{0}^{1}\left(\int_{0}^{\tau} g(s) d s\right)(\tau-1) \varphi_{(x, y)}^{\prime}(\tau) d \tau,
\end{aligned}
$$

which proves the identity in (2.1).

Theorem 2.1. Let $f$ be an operator convex function on $C$ and $x, y \in C$, with $x \neq y$. If $p:[0,1] \rightarrow \mathbb{R}$ is a Lebesgue integrable function such that

$$
0 \leq \int_{0}^{\tau} p(s) d s \leq \int_{0}^{1} p(s) d s \text { for all } \tau \in[0,1],
$$


then we have the inequalities

$$
\begin{aligned}
& \int_{0}^{1}\left(\int_{\tau}^{1} p(s) d s\right) \tau d \tau \nabla_{+} f_{x}(y-x) \\
& -\int_{0}^{1}\left(\int_{0}^{\tau} p(s) d s\right)(1-\tau) d \tau \nabla_{-} f_{y}(y-x) \\
& \leq \int_{0}^{1} p(\tau) f((1-\tau) x+\tau y) d \tau-\int_{0}^{1} p(\tau) d \tau \int_{0}^{1} f((1-\tau) x+\tau y) d \tau \\
& \leq \int_{0}^{1}\left(\int_{\tau}^{1} p(s) d s\right) \tau d \tau \nabla_{-} f_{y}(y-x) \\
& -\int_{0}^{1}\left(\int_{0}^{\tau} p(s) d s\right)(1-\tau) d \tau \nabla_{+} f_{x}(y-x)
\end{aligned}
$$

or, equivalently,

$$
\begin{aligned}
& \int_{0}^{1}(1-\tau)\left(\int_{0}^{\tau}\left[p(1-s) \nabla_{-} f_{y}(y-x)-p(s) \nabla_{+} f_{x}(y-x)\right] d s\right) d \tau \\
& \leq \int_{0}^{1} p(\tau) f((1-\tau) x+\tau y) d \tau-\int_{0}^{1} p(\tau) d \tau \int_{0}^{1} f((1-\tau) x+\tau y) d \tau \\
& \leq \int_{0}^{1}(1-\tau)\left(\int_{0}^{\tau}\left[p(1-s) \nabla_{+} f_{x}(y-x)-p(s) \nabla_{-} f_{y}(y-x)\right] d s\right) d \tau .
\end{aligned}
$$

Proof. We have for $\varphi_{(x, y)}$ and $p:[0,1] \rightarrow \mathbb{R}$ a Lebesgue integrable function that

$$
\begin{aligned}
& \int_{0}^{1} p(\tau) \varphi_{(x, y)}(\tau) d \tau-\int_{0}^{1} p(\tau) d \tau \int_{0}^{1} \varphi_{(x, y)}(\tau) d \tau \\
& =\int_{0}^{1}\left(\int_{\tau}^{1} p(s) d s\right)(\tau) \varphi_{(x, y)}^{\prime}(\tau) d \tau \\
& -\int_{0}^{1}\left(\int_{0}^{\tau} p(s) d s\right)(1-\tau) \varphi_{(x, y)}^{\prime}(\tau) d \tau
\end{aligned}
$$

By the gradient inequalities for $\varphi_{(x, y)}$ we have

$$
\tau \nabla_{-} f_{y}(y-x) \geq \tau \varphi_{(x, y)}^{\prime}(\tau) \geq \tau \nabla_{+} f_{x}(y-x)
$$

and

$$
(1-\tau) \nabla_{-} f_{y}(y-x) \geq(1-\tau) \varphi_{(x, y)}^{\prime}(\tau) \geq(1-\tau) \nabla_{+} f_{x}(y-x)
$$

for all $\tau \in(0,1)$.

From

we get that $\int_{\tau}^{1} p(s) d s \geq 0$ for all $\tau \in(0,1)$.

$$
\int_{0}^{\tau} p(s) d s \leq \int_{0}^{1} p(s) d s=\int_{0}^{\tau} p(s) d s+\int_{\tau}^{1} p(s) d s,
$$

From (2.9) we derive that

$$
\begin{aligned}
\left(\int_{\tau}^{1} p(s) d s\right) \tau \nabla_{-} f_{y}(y-x) & \geq\left(\int_{\tau}^{1} p(s) d s\right) \tau \varphi_{(x, y)}^{\prime}(\tau) \\
& \geq\left(\int_{\tau}^{1} p(s) d s\right) \tau \nabla_{+} f_{x}(y-x)
\end{aligned}
$$


and from (2.10) that

$$
\begin{aligned}
-\left(\int_{0}^{\tau} p(s) d s\right)(1-\tau) \nabla_{+} f_{x}(y-x) & \leq-\left(\int_{0}^{\tau} p(s) d s\right)(1-\tau) \varphi_{(x, y)}^{\prime}(\tau) \\
& \leq-\left(\int_{0}^{\tau} p(s) d s\right)(1-\tau) \nabla_{-} f_{y}(y-x)
\end{aligned}
$$

all $\tau \in(0,1)$.

If we integrate these inequalities over $\tau \in[0,1]$ and add the obtained results, then we get

$$
\begin{aligned}
& \int_{0}^{1}\left(\int_{\tau}^{1} p(s) d s\right) \tau d \tau \nabla_{-} f_{y}(y-x)-\int_{0}^{1}\left(\int_{0}^{\tau} p(s) d s\right)(1-\tau) d \tau \nabla_{+} f_{x}(y-x) \\
& \geq \int_{0}^{1}\left(\int_{\tau}^{1} p(s) d s\right) \tau \varphi_{(x, y)}^{\prime}(\tau) d \tau-\int_{0}^{1}\left(\int_{0}^{\tau} p(s) d s\right)(1-\tau) \varphi_{(x, y)}^{\prime}(\tau) d \tau \\
& \geq \int_{0}^{1}\left(\int_{\tau}^{1} p(s) d s\right) \tau d \tau \nabla_{+} f_{x}(y-x)-\int_{0}^{1}\left(\int_{0}^{\tau} p(s) d s\right)(1-\tau) d \tau \nabla_{-} f_{y}(y-x) .
\end{aligned}
$$

By using the equality (2.1) we obtain

$$
\begin{aligned}
& \int_{0}^{1}\left(\int_{\tau}^{1} p(s) d s\right) \tau d \tau \nabla_{+} f_{x}(y-x) \\
& -\int_{0}^{1}\left(\int_{0}^{\tau} p(s) d s\right)(1-\tau) d \tau \nabla_{-} f_{y}(y-x) \\
& \leq \int_{0}^{1} p(\tau) \varphi_{(x, y)}(\tau) d \tau-\int_{0}^{1} p(\tau) d \tau \int_{0}^{1} \varphi_{(x, y)}(\tau) d \tau \\
& \leq \int_{0}^{1}\left(\int_{\tau}^{1} p(s) d s\right) \tau d \tau \nabla_{-} f_{y}(y-x) \\
& -\int_{0}^{1}\left(\int_{0}^{\tau} p(s) d s\right)(1-\tau) d \tau \nabla_{+} f_{x}(y-x),
\end{aligned}
$$

namely (2.6).

If we change the variable $y=1-\tau$, then we have

$$
\int_{0}^{1}\left(\int_{\tau}^{1} p(s) d s\right) \tau d \tau=\int_{0}^{1}\left(\int_{1-y}^{1} p(s) d s\right)(1-y) d y .
$$

Also by the change of variable $u=1-s$, we get

$$
\int_{1-y}^{1} p(s) d s=\int_{0}^{y} p(1-u) d u
$$

which implies that

$$
\int_{0}^{1}\left(\int_{\tau}^{1} p(s) d s\right) \tau d \tau=\int_{0}^{1}\left(\int_{0}^{\tau} p(1-s) d s\right)(1-\tau) d \tau .
$$


Therefore

$$
\begin{aligned}
& \int_{0}^{1}\left(\int_{\tau}^{1} p(s) d s\right) \tau d \tau \nabla_{-} f_{y}(y-x)-\int_{0}^{1}\left(\int_{0}^{\tau} p(s) d s\right)(1-\tau) d \tau \nabla_{+} f_{x}(y-x) \\
& =\int_{0}^{1}\left(\int_{0}^{\tau} p(1-s) d s\right)(1-\tau) d \tau \nabla_{-} f_{y}(y-x) \\
& -\int_{0}^{1}\left(\int_{0}^{\tau} p(s) d s\right)(1-\tau) d \tau \nabla_{+} f_{x}(y-x) \\
& =\int_{0}^{1}(1-\tau)\left(\int_{0}^{\tau}\left[p(1-s) \nabla_{-} f_{y}(y-x)-p(s) \nabla_{+} f_{x}(y-x)\right] d s\right) d \tau \\
& \text { and } \\
& \int_{0}^{1}\left(\int_{\tau}^{1} p(s) d s\right) \tau d \tau \nabla_{+} f_{x}(y-x)-\int_{0}^{1}\left(\int_{0}^{\tau} p(s) d s\right)(1-\tau) d \tau \nabla_{-} f_{y}(y-x) \\
& =\int_{0}^{1}\left(\int_{0}^{\tau} p(1-s) d s\right)(1-\tau) d \tau \nabla_{+} f_{x}(y-x) \\
& -\int_{0}^{1}\left(\int_{0}^{\tau} p(s) d s\right)(1-\tau) d \tau \nabla_{-} f_{y}(y-x) \\
& =\int_{0}^{1}(1-\tau)\left(\int_{0}^{\tau}\left[p(1-s) \nabla_{+} f_{x}(y-x)-p(s) \nabla_{-} f_{y}(y-x)\right] d s\right) d \tau,
\end{aligned}
$$

and by (2.11) we get (2.7).

We say that the function $p:[0,1] \rightarrow \mathbb{R}$ is symmetric on $[0,1]$ if

$$
p(1-t)=p(t) \text { for all } t \in[0,1] .
$$

Corollary 2.1. Let $f$ be a convex function on $C$ and $x, y \in C$, with $x \neq y$. If $p:[0,1] \rightarrow \mathbb{R}$ is a Lebesgue integrable and symmetric function such that the condition (2.5) holds, then we have

$$
\begin{aligned}
& \left|\frac{1}{\int_{0}^{1} p(\tau) d \tau} \int_{0}^{1} p(\tau) f((1-\tau) x+\tau y) d \tau-\int_{0}^{1} f((1-\tau) x+\tau y) d \tau\right| \\
& \leq \frac{1}{\int_{0}^{1} p(\tau) d \tau} \int_{0}^{1}\left(\int_{0}^{\tau} p(s) d s\right)(1-\tau) d \tau\left[\nabla_{-} f_{y}(y-x)-\nabla_{+} f_{x}(y-x)\right] \\
& \leq \frac{1}{2}\left[\nabla_{-} f_{y}(y-x)-\nabla_{+} f_{x}(y-x)\right] .
\end{aligned}
$$

Proof. Since $p$ is symmetric, then $p(1-s)=p(s)$ for all $s \in[0,1]$ and by $(2.7)$ we get

$$
\begin{aligned}
& \int_{0}^{1}\left(\int_{0}^{\tau} p(s) d s\right)(1-\tau) d \tau\left[\nabla_{+} f_{x}(y-x)-\nabla_{-} f_{y}(y-x)\right] \\
& \leq \int_{0}^{1} p(\tau) \varphi_{(x, y)}(\tau) d \tau-\int_{0}^{1} p(\tau) d \tau \int_{0}^{1} \varphi_{(x, y)}(\tau) d \tau \\
& \leq\left[\nabla_{-} f_{y}(y-x)-\nabla_{+} f_{x}(y-x)\right] \int_{0}^{1}\left(\int_{0}^{\tau} p(s) d s\right)(1-\tau) d \tau,
\end{aligned}
$$

which is equivalent to the first inequality in (2.12). 
Since $0 \leq \int_{0}^{\tau} p(s) d s \leq \int_{0}^{1} p(\tau) d \tau$, hence

$$
\int_{0}^{1}\left(\int_{0}^{\tau} p(s) d s\right)(1-\tau) d \tau \leq \int_{0}^{1} p(\tau) d \tau \int_{0}^{1}(1-\tau) d \tau=\frac{1}{2} \int_{0}^{1} p(\tau) d \tau
$$

and the last part of (2.12) is proved.

Remark 2.1. If the function $p$ is nonnegative and symmetric then the inequality (2.12) holds true.

If we consider the weight $p:[0,1] \rightarrow[0, \infty), p(s)=\left|s-\frac{1}{2}\right|$, then

$$
\begin{aligned}
& \int_{0}^{1}\left(\int_{0}^{\tau} p(s) d s\right)(1-\tau) d \tau \\
& =\int_{0}^{1}\left(\int_{0}^{\tau}\left|s-\frac{1}{2}\right| d s\right)(1-\tau) d \tau \\
& =\int_{0}^{\frac{1}{2}}\left(\int_{0}^{\tau}\left|s-\frac{1}{2}\right| d s\right)(1-\tau) d \tau \\
& +\int_{\frac{1}{2}}^{1}\left(\int_{0}^{\tau}\left|s-\frac{1}{2}\right| d s\right)(1-\tau) d \tau \\
& =\int_{0}^{\frac{1}{2}}\left(\int_{0}^{\tau}\left(\frac{1}{2}-s\right) d s\right)(1-\tau) d \tau \\
& +\int_{\frac{1}{2}}^{1}\left(\int_{0}^{\frac{1}{2}}\left(\frac{1}{2}-s\right) d s+\int_{\frac{1}{2}}^{\tau}\left(s-\frac{1}{2}\right)\right)(1-\tau) d \tau \\
& =\int_{0}^{\frac{1}{2}}\left(\frac{1}{2} \tau-\frac{\tau^{2}}{2}\right)(1-\tau) d \tau \\
& +\int_{\frac{1}{2}}^{1}\left(\int_{0}^{\frac{1}{2}}\left(\frac{1}{2}-s\right) d s+\int_{\frac{1}{2}}^{\tau}\left(s-\frac{1}{2}\right) d s\right)(1-\tau) d \tau .
\end{aligned}
$$

We have

$$
\begin{aligned}
& \int_{0}^{\frac{1}{2}}\left(\frac{1}{2} \tau-\frac{\tau^{2}}{2}\right)(1-\tau) d \tau=\frac{1}{2} \int_{0}^{\frac{1}{2}}(1-\tau) \tau(1-\tau) d \tau \\
& =\frac{1}{2} \int_{0}^{\frac{1}{2}}(1-\tau)^{2} \tau d \tau=\frac{11}{384}
\end{aligned}
$$

and

$$
\begin{aligned}
& \int_{\frac{1}{2}}^{1}\left(\int_{0}^{\frac{1}{2}}\left(\frac{1}{2}-s\right) d s+\int_{\frac{1}{2}}^{\tau}\left(s-\frac{1}{2}\right) d s\right)(1-\tau) d \tau \\
& =\int_{\frac{1}{2}}^{1}\left(\frac{1}{8}+\frac{1}{2}\left(\tau-\frac{1}{2}\right)^{2}\right)(1-\tau) d \tau \\
& =\frac{1}{8} \int_{\frac{1}{2}}^{1}(1-\tau) d \tau+\frac{1}{2} \int_{\frac{1}{2}}^{1}\left(\tau-\frac{1}{2}\right)^{2}(1-\tau) d \tau=\frac{7}{384} .
\end{aligned}
$$


Therefore

$$
\int_{0}^{1}\left(\int_{0}^{\tau} p(s) d s\right)(1-\tau) d \tau=\frac{3}{64}
$$

Since $\int_{0}^{1}\left|\tau-\frac{1}{2}\right| d \tau=\frac{1}{4}$, hence

$$
\frac{1}{\int_{0}^{1} p(\tau) d \tau} \int_{0}^{1}\left(\int_{0}^{\tau} p(s) d s\right)(1-\tau) d \tau=\frac{3}{16} .
$$

Utilising (2.12) for symmetric weight $p:[0,1] \rightarrow[0, \infty), p(s)=\left|s-\frac{1}{2}\right|$, we get

$$
\begin{aligned}
& \left|4 \int_{0}^{1}\right| \tau-\frac{1}{2}\left|f((1-\tau) x+\tau y) d \tau-\int_{0}^{1} f((1-\tau) x+\tau y) d \tau\right| \\
& \leq \frac{3}{16}\left[\nabla_{-} f_{y}(y-x)-\nabla_{+} f_{x}(y-x)\right]
\end{aligned}
$$

where $f$ is a convex function on $C$ and $x, y \in C$, with $x \neq y$.

Consider now the symmetric function $p(s)=(1-s) s, x \in[0,1]$. Then

$$
\int_{0}^{\tau} p(s) d s=\int_{a}^{\tau}(1-s) s d s=-\frac{1}{6} \tau^{2}(2 \tau-3), \tau \in[0,1]
$$

and

$$
\int_{0}^{1}\left(\int_{0}^{\tau} p(s) d s\right)(1-\tau) d \tau=-\frac{1}{6} \int_{0}^{1} \tau^{2}(2 \tau-3)(1-\tau) d \tau=\frac{1}{40} .
$$

Also

$$
\int_{0}^{1} p(\tau) d \tau=\int_{0}^{1}(1-\tau) \tau d \tau=\frac{1}{6}
$$

and

$$
\frac{1}{\int_{0}^{1} p(\tau) d \tau} \int_{0}^{1}\left(\int_{0}^{\tau} p(s) d s\right)(1-\tau) d \tau=\frac{3}{20}
$$

and by (2.12) we obtain

$$
\begin{aligned}
& \left|6 \int_{0}^{1}(1-\tau) \tau f((1-\tau) x+\tau y) d \tau-\int_{0}^{1} f((1-\tau) x+\tau y) d \tau\right| \\
& \leq \frac{3}{20}\left[\nabla_{-} f_{y}(y-x)-\nabla_{+} f_{x}(y-x)\right],
\end{aligned}
$$

where $f$ is a convex function on $C$ and $x, y \in C$, with $x \neq y$.

\section{Examples for Norms}

Now, assume that $(X,\|\cdot\|)$ is a normed linear space. The function $f_{0}(s)=$ $\frac{1}{2}\|x\|^{2}, x \in X$ is convex and thus the following limits exist

(iv) $\langle x, y\rangle_{s}:=\nabla_{+} f_{0, y}(x)=\lim _{t \rightarrow 0+} \frac{\|y+t x\|^{2}-\|y\|^{2}}{2 t}$;

(v) $\langle x, y\rangle_{i}:=\nabla_{-} f_{0, y}(x)=\lim _{s \rightarrow 0-} \frac{\|y+s x\|^{2}-\|y\|^{2}}{2 s}$; 
for any $x, y \in X$. They are called the lower and upper semi-inner products associated to the norm $\|\cdot\|$.

For the sake of completeness we list here some of the main properties of these mappings that will be used in the sequel (see for example [2] or [6]), assuming that $p, q \in\{s, i\}$ and $p \neq q$ :

(a) $\langle x, x\rangle_{p}=\|x\|^{2}$ for all $x \in X$;

(aa) $\langle\alpha x, \beta y\rangle_{p}=\alpha \beta\langle x, y\rangle_{p}$ if $\alpha, \beta \geq 0$ and $x, y \in X$;

(aaa) $\left|\langle x, y\rangle_{p}\right| \leq\|x\|\|y\|$ for all $x, y \in X$;

(av) $\langle\alpha x+y, x\rangle_{p}=\alpha\langle x, x\rangle_{p}+\langle y, x\rangle_{p}$ if $x, y \in X$ and $\alpha \in \mathbb{R}$;

(v) $\langle-x, y\rangle_{p}=-\langle x, y\rangle_{q}$ for all $x, y \in X$;

(va) $\langle x+y, z\rangle_{p} \leq\|x\|\|z\|+\langle y, z\rangle_{p}$ for all $x, y, z \in X$;

(vaa) The mapping $\langle\cdot, \cdot\rangle_{p}$ is continuous and subadditive (superadditive) in the first variable for $p=s \quad$ (or $p=i)$;

(vaaa) The normed linear space $(X,\|\cdot\|)$ is smooth at the point $x_{0} \in X \backslash\{0\}$ if and only if $\left\langle y, x_{0}\right\rangle_{s}=\left\langle y, x_{0}\right\rangle_{i}$ for all $y \in X$; in general $\langle y, x\rangle_{i} \leq\langle y, x\rangle_{s}$ for all $x, y \in X$

(ax) If the norm $\|\cdot\|$ is induced by an inner product $\langle\cdot, \cdot\rangle$, then $\langle y, x\rangle_{i}=\langle y, x\rangle=$ $\langle y, x\rangle_{s}$ for all $x, y \in X$.

The function $f_{r}(x)=\|x\|^{r}(x \in X$ and $1 \leq r<\infty)$ is also convex. Therefore, the following limits, which are related to the superior (inferior) semi-inner products,

$$
\begin{aligned}
\nabla_{ \pm} f_{r, y}(x) & :=\lim _{t \rightarrow 0 \pm} \frac{\|y+t x\|^{r}-\|y\|^{r}}{t} \\
& =r\|y\|^{r-1} \lim _{t \rightarrow 0 \pm} \frac{\|y+t x\|-\|y\|}{t}=r\|y\|^{r-2}\langle x, y\rangle_{s(i)}
\end{aligned}
$$

exist for all $x, y \in X$ whenever $r \geq 2$; otherwise, they exist for any $x \in X$ and nonzero $y \in X$. In particular, if $r=1$, then the following limits

$$
\nabla_{ \pm} f_{1, y}(x):=\lim _{t \rightarrow 0 \pm} \frac{\|y+t x\|-\|y\|}{t}=\frac{\langle x, y\rangle_{s(i)}}{\|y\|}
$$

exist for $x, y \in X$ and $y \neq 0$.

If $p:[0,1] \rightarrow \mathbb{R}$ is a Lebesgue integrable and symmetric function such that the condition

$$
0 \leq \int_{0}^{\tau} p(s) d s \leq \int_{0}^{1} p(s) d s \text { for all } \tau \in[0,1],
$$

is valid, then by (2.12) we get

$$
\begin{aligned}
& \left|\frac{1}{\int_{0}^{1} p(\tau) d \tau} \int_{0}^{1} p(\tau)\|(1-\tau) x+\tau y\|^{r} d \tau-\int_{0}^{1}\|(1-\tau) x+\tau y\|^{r} d \tau\right| \\
& \leq \frac{r}{\int_{0}^{1} p(\tau) d \tau} \int_{0}^{1}\left(\int_{0}^{\tau} p(s) d s\right)(1-\tau) d \tau \\
& \times\left[\|y\|^{r-2}\langle y-x, y\rangle_{i}-\|x\|^{r-2}\langle y-x, x\rangle_{s}\right] .
\end{aligned}
$$

If $r \geq 2$, then the inequality (3.1) holds for all $x, y \in X$. If $r \in[1,2)$, then the inequality (3.1) holds for all $x, y \in X$ with $x, y \neq 0$. 
For $r=2$ we get

$$
\begin{aligned}
& \left|\frac{1}{\int_{0}^{1} p(\tau) d \tau} \int_{0}^{1} p(\tau)\|(1-\tau) x+\tau y\|^{2} d \tau-\int_{0}^{1}\|(1-\tau) x+\tau y\|^{2} d \tau\right| \\
& \leq \frac{2}{\int_{0}^{1} p(\tau) d \tau} \int_{0}^{1}\left(\int_{0}^{\tau} p(s) d s\right)(1-\tau) d \tau\left[\langle y-x, y\rangle_{i}-\langle y-x, x\rangle_{s}\right]
\end{aligned}
$$

for all $x, y \in X$.

If we take $p(\tau)=\left|\tau-\frac{1}{2}\right|, \tau \in[0,1]$ in (3.1), then we obtain

$$
\begin{aligned}
& \left|4 \int_{0}^{1}\right| \tau-\frac{1}{2}\left|\|(1-\tau) x+\tau y\|^{r} d \tau-\int_{0}^{1}\|(1-\tau) x+\tau y\|^{r} d \tau\right| \\
& \leq \frac{3 r}{16}\left[\|y\|^{r-2}\langle y-x, y\rangle_{i}-\|x\|^{r-2}\langle y-x, x\rangle_{s}\right] .
\end{aligned}
$$

If $X=H$ a real inner product space, then from (3.2) we get

$$
\begin{aligned}
& \left|\frac{1}{\int_{0}^{1} p(\tau) d \tau} \int_{0}^{1} p(\tau)\|(1-\tau) x+\tau y\|^{2} d \tau-\int_{0}^{1}\|(1-\tau) x+\tau y\|^{2} d \tau\right| \\
& \leq \frac{2}{\int_{0}^{1} p(\tau) d \tau} \int_{0}^{1}\left(\int_{0}^{\tau} p(s) d s\right)(1-\tau) d \tau\|y-x\|^{2}
\end{aligned}
$$

for all $x, y \in H$.

\section{Examples for Functions of Several Variables}

Now, let $\Omega \subset \mathbb{R}^{n}$ be an open convex set in $\mathbb{R}^{n}$. If $F: \Omega \rightarrow \mathbb{R}$ is a differentiable convex function on $\Omega$, then, obviously, for any $\bar{c} \in \Omega$ we have

$$
\nabla F_{\bar{c}}(\bar{y})=\sum_{i=1}^{n} \frac{\partial F(\bar{c})}{\partial x_{i}} \cdot y_{i}, \bar{y}=\left(y_{1}, \ldots, y_{n}\right) \in \mathbb{R}^{n},
$$

where $\frac{\partial F}{\partial x_{i}}$ are the partial derivatives of $F$ with respect to the variable $x_{i}(i=$ $1, \ldots, n)$.

If $p:[0,1] \rightarrow \mathbb{R}$ is a Lebesgue integrable and symmetric function such that the condition (2.5) holds, then we have for all $\bar{a}, \bar{b} \in \Omega$ that

$$
\begin{aligned}
& \left|\frac{1}{\int_{0}^{1} p(\tau) d \tau} \int_{0}^{1} p(\tau) F((1-\tau) \bar{a}+\tau \bar{b}) d \tau-\int_{0}^{1} f((1-\tau) \bar{a}+\tau \bar{b}) d \tau\right| \\
& \leq \frac{1}{\int_{0}^{1} p(\tau) d \tau} \int_{0}^{1}\left(\int_{0}^{\tau} p(s) d s\right)(1-\tau) d \tau \\
& \times \sum_{i=1}^{n}\left(\frac{\partial F(\bar{b})}{\partial x_{i}}-\frac{\partial F(\bar{a})}{\partial x_{i}}\right)\left(b_{i}-a_{i}\right) \\
& \leq \frac{1}{2} \sum_{i=1}^{n}\left(\frac{\partial F(\bar{b})}{\partial x_{i}}-\frac{\partial F(\bar{a})}{\partial x_{i}}\right)\left(b_{i}-a_{i}\right) .
\end{aligned}
$$


If we take $p(\tau)=\left|\tau-\frac{1}{2}\right|, \tau \in[0,1]$ in (4.1), then we get

$$
\begin{aligned}
& \left|4 \int_{0}^{1}\right| \tau-\frac{1}{2}\left|F((1-\tau) \bar{a}+\tau \bar{b}) d \tau-\int_{0}^{1} f((1-\tau) \bar{a}+\tau \bar{b}) d \tau\right| \\
& \leq \frac{3}{16} \sum_{i=1}^{n}\left(\frac{\partial F(\bar{b})}{\partial x_{i}}-\frac{\partial F(\bar{a})}{\partial x_{i}}\right)\left(b_{i}-a_{i}\right)
\end{aligned}
$$

for all $\bar{a}, \bar{b} \in \Omega$.

\section{References}

[1] M. Alomari and M. Darus, Refinements of s-Orlicz convex functions in normed linear spaces. Int. J. Contemp. Math. Sci. 3 (2008), no. 29-32, 1569-1594.

[2] I. Ciorănescu, Geometry of Banach Spaces, Duality Mappings and Nonlinear Problems, Kluwer Academic Publishers, Dordrecht, 1990.

[3] X. Chen, New convex functions in linear spaces and Jensen's discrete inequality. $J$. Inequal. Appl. 2013, 2013:472, 8 pp.

[4] S. S. Dragomir, An inequality improving the first Hermite-Hadamard inequality for convex functions defined on linear spaces and applications for semi-inner products, J. Inequal. Pure 85 Appl. Math. 3 (2002), no. 2, Article 31, 8 pp.

[5] S. S. Dragomir, An inequality improving the second Hermite-Hadamard inequality for convex functions defined on linear spaces and applications for semi-inner products, J. Inequal. Pure $\mathscr{B}$ Appl. Math. 3 (2002), no. 3, Article 35, 8 pp.

[6] S. S. Dragomir, Semi-inner Products and Applications. Nova Science Publishers, Inc., Hauppauge, NY, 2004. x+222 pp. ISBN: 1-59033-947-9

[7] S. S. Dragomir, Refinements and reverses of Féjer's inequalities for convex functions on linear spaces, Preprint RGMIA Res. Rep. Coll. 22 (2019), Art. 92, 14 pp. [Online http://rgmia.org/papers/v22/v22a92.pdf] .

[8] S. S. Dragomir and C. E. M. Pearce, Selected Topics on Hermite-Hadamard Inequalities and Applications, RGMIA Monographs, Victoria University, 2000. [Online https://rgmia.org/monographs/hermite_hadamard.html].

[9] M. Adil Khan, S. Khalid and J. Pečarić, Improvement of Jensen's inequality in terms of Gâteaux derivatives for convex functions in linear spaces with applications. Kyungpook Math. J. 52 (2012), no. 4, 495-511.

[10] E. Kikianty and S. S. Dragomir, Hermite-Hadamard's inequality and the $p-H H$ norm on the Cartesian product of two copies of a normed space. Math. Inequal. Appl. 13 (2010), no. 1, 1-32.

[11] E. Kikianty, S. S. Dragomir and P. Cerone, Ostrowski type inequality for absolutely continuous functions on segments in linear spaces. Bull. Korean Math. Soc. 45 (2008), no. 4, 763-780.

[12] E. Kikianty, S. S. Dragomir and P. Cerone, Sharp inequalities of Ostrowski type for convex functions defined on linear spaces and application. Comput. Math. Appl. 56 (2008), no. 9, 2235-2246.

Silvestru Sever Dragomir

Mathematics, College of Engineering $\mathcal{G}$ Science, Victoria University, PO Box 14428, Melbourne City, MC 8001, Australia 
DST-NRF Centre of Excellence in the Mathematical, and Statistical Sciences, School of Computer Science, \&3 Applied Mathematics, University of the Witwatersrand,, Private Bag 3, Johannesburg 2050, South Africa

E-mail address: sever.dragomir@vu.edu.au

Received: December 11, 2019; Accepted: July 27, 2020 